Pacific Journal of Mathematics

NOTES ON THE FEYNMAN INTEGRAL.

Wiujam Johnson and David Le STout 


\title{
NOTES ON THE FEYNMAN INTEGRAL, I
}

\author{
G. W. Johnson AND D. L. Skoug
}

We extend somewhat and simplify substantially some of the recent work of Cameron and Storvick involving the analytic Feynman integral of certain functions on Wiener space of the form $F(x)=\exp \left\{\int_{a}^{b} \theta(t, x(t)) d t\right\}$; here $\theta$ is a complexvalued function on $(a, b] \times R$ and $x$ is an element of Wiener space, that is, a continuous function on $[a, b]$ which vanishes at $a$.

1. Introduction. In a recent paper [2], Cameron and Storvick treat a Banach algebra $S$ of functions on Wiener space which are a kind of stochastic Fourier transform of Borel measures on $L_{2}[a, b]$. (Precise definitions will be given in the next section.) For such functions they show that the analytic Feynman integral, defined by analytic continuation of the Wiener integral, exists, and they give a formula for this Feynman integral. The work in [2] is related to Albeverio and Høegh-Krohn's beautiful theory [1] of infinite dimensional oscillatory integrals ("Fresnel integrals") as well as to [5]. Cameron and Storvick's work is highly promising and has some appealing features. For example, as we will show in a later note, the existence of the Feynman integral for certain qudratic potentials can be established without having to construct special spaces, quadratic forms, etc. to fit the particular problem of interest.

The main purpose of this note is to show that a crucial part of [2] can be substantially simplified. Let $\boldsymbol{R}, \boldsymbol{C}$ denote the real and complex numbers respectively. Let $\theta$ map $(a, b] \times \boldsymbol{R}$ to $\boldsymbol{C}$. Let $C[a, b]$ denote Wiener space; that is, the space of $\boldsymbol{R}$-valued continuous functions on $[a, b]$ which vanish at $a$. Let $m$ denote Wiener measure on $C[a, b]$. Under certain hypotheses on $\theta$, Cameron and Storvick show that the function

$$
F(x)=\exp \left\{\int_{a}^{b} \theta(t, x(t)) d t\right\}, x \text { in } C[a, b],
$$

belongs to the Banach algebra $S$ and hence possesses an analytic Feynman integral. This result depends on some rather elaborate machinery; for example, their spaces $\mathscr{C}^{\prime}, S^{\prime}, \mathscr{C}^{\prime \prime}, S^{\prime \prime}, \mathscr{M}_{n}^{\prime \prime}, S_{n}^{\prime \prime}$, $S_{n}^{\wedge}, \mathscr{M}_{n}^{\wedge}$ are all part of this picture. We give a simpler proof of this result avoiding the machinery. We also extend their result somewhat, but it is the simplification that is the main point. It should be mentioned that the results on $\mathscr{M}^{\prime}, S^{\prime}, \mathscr{L}^{\prime \prime}$, etc. are 
interesting in their own right and may well prove useful in identifying other functions in the space $S$ and in other ways. However, it is functionals of the form (1.1) that are of primary interest with regard to the physical motivation in quantum mechanics.

[2] deals throughout with functions $F$ on $C^{\nu}[a, b]$. We will restrict attention to the case $\nu=1$ for notational simplicity, but our arguments work just as well for general $\nu$.

2. Preliminaries; Some simple results and comments. A subset $A$ of Wiener space is said to be scale-invariant measurable provided $\rho A$ is Wiener measurable for every $\rho>0$. A scale-invariant measurable set $N$ is said to be scale-invariant null provided $m(\rho N)=0$ for every $\rho>0$. A property which holds except on a scale-invariant null set is said to hold scale-invariant almost everywhere $(s-$ a.e.). The class of scale-invariant measurable sets form a $\sigma$-algebra [4]. A function $F$ on $C[a, b]$ is said to be scale-invariant measurable if it is measurable with respect to this $\sigma$-algebra. We begin with the definition of the analytic Feynman integral.

Let $F$ be a function which is scale-invariant measurable and $s$-a.e. defined and which is such that the Wiener integral

$$
J(\lambda)=\int_{C[a, b]} F\left(\lambda^{-1 / 2} x\right) d m(x)
$$

exists for all $\lambda>0$. If there exists a function $J^{*}(\lambda)$ analytic in $C^{+}=\{\lambda$ in $C: \operatorname{Re} \lambda>0\}$ such that $J^{*}(\lambda)=J(\lambda)$ for all $\lambda>0$, then $J^{*}(\lambda)$ is defined to be the analytic Wiener integral of $F$ over $C[a$, $b]$ with parameter $\lambda$, and, for $\lambda$ in $\boldsymbol{C}^{+}$, we write

$$
\int_{C[a, b]}^{a n w_{\lambda}} F(x) d m(x) \equiv J^{*}(\lambda) .
$$

Let $q$ be a real parameter $(q \neq 0)$ and let $F$ be a function whose analytic Wiener integral exists for $\lambda$ in $C^{+}$. If the following limit exists, we call it the analytic Feynman integral of $F$ over $C[a, b]$ with parameter $q$ and we write

$$
\int_{C[a b]}^{\alpha n f_{q}} F(x) d m(x) \equiv \lim _{\lambda \rightarrow-i q} \int_{C[a, b]}^{a n w \lambda} F(x) d m(x)
$$

where $\lambda$ approaches $-i q$ through $C^{+}$.

REMARK. Equality $s$-a.e. is an equivalence relation. It is the appropriate equivalence relation for the analytic Feynman integral. One can, for example, find functions $F$ and $G$ on $C[a, b]$ which are Borel measurable and equal $m$-a.e. but such that the analytic Feynman integral of $F$ exists but the analytic Feynman integral of 
$G$ does not exist. A detailed discussion of topics related to scaleinvariant measurability can be found in [4].

Let $L_{2}=L_{2}[a, b]$ denote as usual the space of Lebesgue measurable, $\boldsymbol{R}$-valued, square-integrable functions on $[a, b]$. Throughout [2], Cameron and Storvick work with the $\sigma$-algebra $\mathscr{A}$ of subsets of $L_{2}$ generated by sets of the form $\left\{v\right.$ in $\left.L_{2}: \int_{a}^{b} v(t) \phi(t) d t<r\right\}$ where $\phi$ is in $L_{2}$ and $r$ is in $\boldsymbol{R}$. Since $L_{2}$ is a separable Banach space, $\mathscr{A}$ is actually just the Borel class of $L_{2}$, that is, the $\sigma$-algebra $\mathscr{B}\left(L_{2}\right)$ generated by the norm-open subsets of $L_{2}$. This fact seems to be quite well known, and one finds it stated in a variety of places, for example [6; p. 115]. The fact that $\mathscr{A}=\mathscr{B}\left(L_{2}\right)$ allows one to simplify some of the arguments in [2] and will be helpful to us in this paper.

The definition of the Banach algebra $S$ with which we will be concerned throughout involves the Paley-Wiener-Zygmund (P. W. Z.) integral [7], a type of stochastic integral which we now define.

Let $\left\{\phi_{j}\right\}$ be a complete orthonormal set of $\boldsymbol{R}$-valued functions of bounded variation on $[a, b]$. For $v$ in $L_{2}[a, b]$, let

$$
v_{n}(t)=\sum_{j=1}^{n}\left[\int_{a}^{b} v(s) \phi_{j}(s) d s\right] \phi_{j}(t) .
$$

The P. W. Z. integral is defined by

$$
\int_{a}^{b} v(s) \tilde{d} x(s) \equiv \lim _{n \rightarrow \infty} \int_{a}^{b} v_{n}(s) d x(s)
$$

for all $x$ in $C[a, b]$ for which the limit exists. It can be shown [7] that this integral exists for $m$-a.e. $x$ and that it is essentially independent of the choice of the sequence $\left\{\phi_{n}\right\}$; further, if $v$ is of bounded variation, the P.W.Z. integral is $m$-a.e. equal to the Riemann-Stieltjes integral $\int_{a}^{b} v(s) d x(s)$.

Now let $\mathscr{C}=\mathscr{M}\left(L_{2}\right)$ be the collection of $C$-valued countably additive measures on $\mathscr{B}\left(L_{2}\right)$. $\mathscr{C}$ is a Banach algebra under the total variation norm where convolution is taken as the multiplication in $\mathscr{C}$.

The Banach algebra $S$ consists of functions $F$ expressible in the form

$$
F(x)=\int_{L_{2}} \exp \left\{i \int_{a}^{b} v(t) \tilde{d} x(t)\right\} d \sigma(v)
$$

for $s$-a.e. $x$ in $C[a, b]$ where $\sigma$ is an element of $\mathscr{M}$. Cameron and Storvick show that the correspondence $\sigma \rightarrow F$ is one-to-one [2; Theorem 2.1] and carries convolution into pointwise multiplication. Letting $\|F\|=\|\sigma\|$ we have that $S$ is a Banach algebra of func- 
tions on Wiener space. Cameron and Storvick show that the analytic Feynman integral exists for every $F$ in $S$. Further they show that if $\left\{F_{j}\right\}$ is a sequence of elements from $S$ such that $\sum_{j=1}^{\infty}\left\|F_{j}\right\|<\infty$, then $F \equiv \sum_{j=1}^{\infty} F_{j}$ is in $S$ and the analytic Feynman integral of $F$ is the sum of the analytic Feynman integrals of the $F_{j}$ 's [2; Theorem 5.4]. Some further results about $S$ are established in [2], but we have summarized the main facts that we will need.

REMARK. The $s$-a.e. equivalence is needed in the definition of $S$. For example, the theorem that every $F$ in $S$ has an analytic Feynman integral would be false if one tried to use the usual $m$ a.e. equivalence.

Cameron and Storvick introduce another Banach algebra $S^{\prime}$ of functions on $C[a, b]$ and they show that $S^{\prime} \subset S$ [2; Theorem 3.0]. They ask whether $S^{\prime}$ is a proper subset of $S$. We finish this section by showing that it is.

Let $B V=B V[a, b]$ be the space of $R$-valued, right continuous functions of bounded variation on $[a, b]$ that vanish at $b$. We consider $B V$ as a subset of $L_{2}$. Hence the Borel class $\mathscr{B}(B V)$ of $B V$ is just $\mathscr{B}\left(L_{2}\right) \cap B V$. Let $\mathscr{C}^{\prime}$ be the class of $C$-valued countably additive measures on $\mathscr{B}(B V)$ and regard $\mathscr{C}^{\prime}$ as equipped with the total variation norm. $S^{\prime}$ consists of functions $F$ expressible in the form

$$
F(x)=\int_{B V} \exp \left\{i \int_{a}^{b} v(t) d x(t)\right\} d \sigma^{\prime}(v)
$$

for s-a.e. $x$ where $\sigma^{\prime}$ is an element of $\mathscr{C}^{\prime}$. Given $\sigma^{\prime}$ in $\mathscr{C}^{\prime}$, define $I \sigma^{\prime}=\sigma$ as follows: $\sigma(E)=\sigma^{\prime}(E \cap B V)$ where $E$ is in $\mathscr{B}\left(L_{2}\right)$. It is easy to check that $I$ imbeds $\mathscr{M}^{\prime}$ in $\mathscr{M}$ and that the question as to whether $S^{\prime}$ is a proper subset of $S$ is equivalent to the question as to whether $I \mathscr{C}^{\prime}$ is a proper subset of $\mathscr{M}$. It is not hard to show that it is; we include the rather simple proof of this result.

Proposition 1. Let $\mathscr{M}_{1} \equiv\left\{\sigma\right.$ in $\mathscr{M}: E, F$ in $\mathscr{B}\left(L_{2}\right)$ and $E \cap$ $B V=F \cap B V$ implies $\sigma(E)=\sigma(F)\} . \quad$ Then $I \mathscr{C l}^{\prime}=\mathscr{C}_{1}$.

Proof. It is clear from the definition of $I$ that $I \mathscr{C}^{\prime} \subset \mathscr{M}_{1}$. Let $\sigma$ be in $\mathscr{A}_{1}$. Define $\sigma^{\prime}$ on $\mathscr{B}(B V)=\mathscr{B}\left(L_{2}\right) \cap B V$ by $\sigma^{\prime}(E \cap B V)=$ $\sigma(E) . \quad \sigma^{\prime}$ is well-defined by definition of $\mathscr{L}_{1}$. Now we show that $\sigma^{\prime}$ is countably additive. Suppose $E_{1} \cap B V, \cdots, E_{n} \cap B V, \cdots$ is a pairwise disjoint sequence from $\mathscr{B}(B V)$. Then

$$
\sigma^{\prime}\left(\bigcup_{n=1}^{\infty}\left(E_{n} \cap B V\right)\right)=\sigma^{\prime}\left(\left(\bigcup_{n=1}^{\infty} E_{n}\right) \cap B V\right)=\sigma\left(\bigcup_{n=1}^{\infty} E_{n}\right)
$$




$$
\begin{aligned}
= & \sigma\left(E_{1} \cup\left(E_{2} \backslash E_{1}\right) \cup \cdots \cup\left(E_{n} \backslash\left(E_{1} \cup \cdots \cup E_{n-1}\right)\right) \cup \cdots\right) \\
= & \sigma\left(E_{1}\right)+\sigma\left(E_{2} \backslash E_{1}\right)+\cdots+\sigma\left(E_{n} \backslash\left(E_{1} \cup \cdots \cup E_{n-1}\right)\right)+\cdots \\
= & \sigma^{\prime}\left(E_{1} \cap B V\right)+\sigma^{\prime}\left(\left(E_{2} \backslash E_{1}\right) \cap B V\right) \\
& \quad+\cdots+\sigma^{\prime}\left(\left(E_{n} \backslash\left(E_{1} \cup \cdots \cup E_{n-1}\right)\right) \cap B V\right)+\cdots \\
= & \sigma^{\prime}\left(E_{1} \cap B V\right)+\sigma^{\prime}\left(E_{2} \cap B V\right)+\cdots+\sigma^{\prime}\left(E_{n} \cap B V\right)+\cdots
\end{aligned}
$$

where the last equality holds since $\left(E_{n} \backslash\left(E_{1} \cup \cdots \cup E_{n-1}\right)\right) \cap B V=E_{n} \cap$ $B V$. Thus $\sigma^{\prime}$, defined as above, is in $\mathscr{C}^{\prime}$. Finally $\left(I \sigma^{\prime}\right)(E)=\sigma^{\prime}(E \cap$ $B V)=\sigma(E)$ and so $\sigma$ is in $I \mathscr{C}^{\prime}$ as desired.

\section{Proposition 2. $I \mathscr{C}^{\prime}=\mathscr{A}_{1} \varsubsetneqq \mathscr{M}$ and so $S^{\prime} \varsubsetneqq S$.}

Proof. Let $\sigma$ be the unit mass concentrated at an element $v_{0}$ of $L_{2} \backslash B V . \quad \sigma$ is in $\mathscr{C}$ clearly. To see that $\sigma$ is not in $\mathscr{C}_{1}$, let $v_{1}$ be another element of $L_{2} \backslash B V$. Then the singleton sets $E=\left\{v_{0}\right\}$ and $F=\left\{v_{1}\right\}$ have the property that $E \cap B V=F \cap B V$ but $\sigma(E) \neq \sigma(F)$.

Is $B V$ in $\mathscr{B}\left(L_{2}\right)$ ? This question is open as far as we know.

The Banach algebra $S^{\prime}$ will not concern us throughout the rest of this paper.

3. Proof that $\exp \left\{\int_{a}^{b} \theta(t, x(t)) d t\right\}$ is in $S$. We begin by considering the following map from $(a, b] \times \boldsymbol{R}$ into $L_{2}[a, b]$.

$$
\Phi(t, v)(s)=\left\{\begin{array}{l}
v, a \leqq s<t \\
0, t \leqq s \leqq b
\end{array}\right.
$$

$\Phi$ is easily seen to be continuous and so is Borel measurable. We begin with two easy measurability lemmas.

LEMma 1. $\int_{a}^{b} \Phi(t, v)(s) \tilde{d} x(s)$ is a Broel measurable function of ( $t$, $v, x)$ on $(a, b] \times \underset{R}{\boldsymbol{R}} \times C[a, b]$. Further, for any Borel measure $\mu$ on $(a, b] \times R, \int_{a}^{b} \Phi(t, v)(s) \tilde{d} x(s)$ is defined except on a $\mu \times m$-null Borel set.

Proof. Let $\left\{\phi_{n}\right\}$ be a complete orthnormal set of functions of bounded variation on $[a, b]$ in terms of which the P.W.Z. integral is defined. Now

$$
\begin{aligned}
& {\left[\int_{a}^{b} \Phi(t, v)(s) \phi_{n}(s) d s\right] \int_{a}^{b} \phi_{n}(s) d x(s)} \\
& \quad=\left[v \int_{a}^{t} \phi_{n}(s) d s\right]\left[\phi_{n}(b) x(b)-\int_{a}^{b} x(s) d \dot{\phi}_{n}(s)\right] .
\end{aligned}
$$

Thus the left hand side of (3.2) is a continuous and hence Borel 
measurable function of $(t, v, x)$. Since $\int_{a}^{b} \Phi(t, v)(s) \tilde{d} x(s)$ is defined as

$$
\lim _{N \rightarrow \infty} \sum_{n=1}^{N}\left[\int_{a}^{b} \Phi(t, v)(s) \phi_{n}(s) d s\right] \int_{a}^{b} \phi_{n}(s) d x(s)
$$

whenever this limit exists, we see that $\int_{a}^{b} \Phi(t, v)(s) \tilde{d} x(s)$ is a Borel measurable function of $(t, v, x)$.

As noted earlier, for every $\psi$ in $L_{2}[a, b], \int_{a}^{b} \psi(s) \widetilde{d} x(s)$ exists for $m$-a.e. $x$. Hence for every $(t, v), \int_{a}^{b} \Phi(t, v)(s) \tilde{d} x(s)$ exists for $m$-a.e. $x$. Now let a Borel measure $\mu$ on $(a, b] \times \boldsymbol{R}$ be given. Then clearly $\int_{a}^{b} \Phi(t, v)(s) \widetilde{d} x(s)$ is defined except on a $\mu \times m$-null Borel set in $(a, b] \times$ $\stackrel{\boldsymbol{R}}{\times} C[a, b]$.

LEMMA 2. Let $\mu$ be any Borel measure on $(a, b] \times \boldsymbol{R}$. Then the Riemann-Stieltjes integral $\int_{a}^{b} \Phi(t, v)(s) d x(s)$ and the P. W. Z. integral $\int_{a}^{b} \Phi(t, v)(s) \tilde{d} x(s)$ are equal except on a $\mu \times m$-null Borel set in $(a, b] \times{ }^{a} \boldsymbol{R} \times C[a, b]$. Hence for $m$-a.e. $x$ they are equal except on a $\mu$-null Borel set in $(a, b] \times \boldsymbol{R}$.

Proof. $\int_{a}^{b} \Phi(t, v)(s) \tilde{d} x(s)$ is Borel measurable by Lemma 1 , and $\int_{a}^{b} \Phi(t, v)(s) d x(s)=v x(t)$ is clearly Borel measurable, and so, the set where they are unequal is a Borel set. Now $\int_{a}^{b} \psi(s) d x(s)=\int_{a}^{b} \psi(s) \widetilde{d} x(s)$ for $m$-a.e. $x$ for any function $\psi$ of bounded variation. Hence for every $(t, v), \int_{a}^{b} \Phi(t, v)(s) d x(s)=\int_{a}^{b} \Phi(t, v)(s) \widetilde{d} x(s)$ for $m$-a.e. $x$. The result follows.

The next lemma is a key step.

Lemma 3. Let $\mu$ be a Borel measure on $(a, b] \times \boldsymbol{R}$. Define $G$ on $C[a, b]$ by

$$
G(x)=\int_{(a, b] \times R} \exp \{i v x(t)\} d \mu(t, v) .
$$

Then $G$ is in $S$.

Proof. We need a measure $\sigma$ on $\mathscr{B}\left(L_{2}\right)$ such that for every $\rho>0$

$$
G(\rho x)=\int_{L_{2}} \exp \left\{i \int_{a} u(s) \widetilde{d} \rho x(s)\right\} d \sigma(u) \text { for } m \text {-a.e. } x
$$


We claim that the Borel measure $\sigma=\mu_{\circ} \Phi^{-1}$ works.

The proof of Lemma 2.1 of [2] shows that $\int_{a}^{b} u(s) \widetilde{d} x(s)$ is a $\mathscr{B}\left(L_{2}\right) \times \mathscr{B}(C[a, b])$ measurable function of $(u, x)$ which is $\sigma \times m$-a.e. defined. Using this fact, the trivial fact that constants involved in the integrator can be taken outside of both the Riemann-Stieltjes and P.W.Z. integrals, the change of variables theorem [3; p. 163] and Lemma 2, we can write

$$
\begin{aligned}
\int_{L_{2}} \cdot \exp & \left\{i \int_{a}^{b} u(s) \tilde{d} \rho x(s)\right\} d \sigma(u) \\
= & \int_{L_{2}} \exp \left\{i \rho \int_{a}^{b} u(s) \widetilde{d} x(s)\right\} d \sigma(u) \\
= & \int_{(a, b] \times \boldsymbol{R}} \exp \left\{i \rho \int_{a}^{b} \Phi(t, v)(s) \tilde{d} x(s)\right\} d \mu(t, v) \\
= & \int_{(a, b] \times \boldsymbol{R}} \exp \left\{i \rho \int_{a}^{b} \Phi(t, v)(s) d x(s)\right\} d \mu(t, v) \\
= & \int_{(a, b] \times \mathbf{R}} \exp \{i \rho v x(t)\} d \mu(t, v)=G(\rho x) \text { as desired }
\end{aligned}
$$

Next we give the main result.

THEOREM 1. Let $\theta:(a, b] \times \boldsymbol{R} \rightarrow \boldsymbol{C}$ be a function which for each $t$ in $(a, b]$ is the Fourier-Stieltjes transform of a $C$-valued countably additive Borel measure $\sigma_{t}$ on $\boldsymbol{R}$; that is,

$$
\theta(t, u)=\int_{R} \exp \{i u v\} d \sigma_{t}(v) .
$$

We assume that $\left\|\sigma_{t}\right\|$ is dominated by a function $h(t)$ in $L_{1}[a, b]$ and that, for each Borel set $E$ in $(a, b] \times \boldsymbol{R}, \sigma_{t}\left(E^{(t)}\right)$ is a Borel measurable function of $t$. (Here $E^{(t)}$ denotes the $t$-section of $E$.) Under these hypotheses, the function defined by (1.1) is in $S$.

REMARK. Cameron and Storvick make the stronger assumption that $\left\|\sigma_{t}\right\|$ is bounded as a function of $t$ rather than dominated by an $L_{1}$-function. Except for this, our assumptions on $\theta$ coincide with theirs.

Proof. Since $S$ is a Banach algebra, it suffices to show that the function

$$
f(x)=\int_{a}^{b} \theta(t, x(t)) d t
$$

is in $S$. This will follow from Lemma 3 if we show that $f$ can be written in the form (3.3). 
For $E$ in $\mathscr{B}((a, b] \times R)$, let $\mu(E)=\int_{a}^{b} \sigma_{t}\left(E^{(t)}\right) d t$. We claim that $\mu$ is a Borel measure on $(a, b] \times R$ with $\|\mu\| \leqq\|h\|_{1}$. Let $\left\{E_{n}\right\}$ be a disjoint sequence of Borel sets from $(a, b] \times \boldsymbol{R}$. For each integer $N, \sum_{n=1}^{N}\left|\sigma_{t}\left(E_{n}^{(t)}\right)\right| \leqq\left\|\sigma_{t}\right\| \leqq h(t)$. Hence, by the Dominated Convergence Theorem,

$$
\begin{aligned}
\mu\left(\bigcup_{n=1}^{\infty} E_{n}\right) & =\int_{a}^{b} \sigma_{t}\left(\bigcup_{n=1}^{\infty} E_{n}^{(t)}\right) d t=\int_{a}^{b} \sum_{n=1}^{\infty} \sigma_{t}\left(E_{n}^{(t)}\right) d t \\
& =\sum_{n=1}^{\infty} \int_{a}^{b} \sigma_{t}\left(E_{n}^{(t)}\right) d t=\sum_{n=1}^{\infty} \mu\left(E_{n}\right),
\end{aligned}
$$

and so $\mu$ is countably additive.

Next we show that $\|\mu\| \leqq\|h\|_{1}$. Let $E_{1}, \cdots, E_{n}$ be any finite sequence of disjoint Borel sets. It suffices to show that $\sum_{j=1}^{n}\left|\mu\left(E_{j}\right)\right| \leqq$ $\|h\|_{1}$. Since $\sum_{j=1}^{n}\left|\sigma_{t}\left(E_{j}^{(t)}\right)\right| \leqq\left\|\sigma_{t}\right\| \leqq h(t)$, we can write

$$
\begin{aligned}
\sum_{j=1}^{n}\left|\mu\left(E_{j}\right)\right| & =\sum_{j=1}^{n}\left|\int_{a}^{b} \sigma_{t}\left(E_{j}^{(t)}\right) d t\right| \leqq \sum_{j=1}^{n} \int_{a}^{b}\left|\sigma_{t}\left(E_{j}^{(t)}\right)\right| d t \\
& =\int_{a}^{b} \sum_{j=1}^{n}\left|\sigma_{t}\left(E_{j}^{(t)}\right)\right| d t \leqq\|h\|_{1} .
\end{aligned}
$$

To finish the proof, it suffices to show that

$$
f(x)=\int_{(a, b] \times \boldsymbol{R}} \exp \{i v x(t)\} d \mu(t, v) .
$$

In fact, we will show that for any bounded Borel measurable function $\phi$ on $(a, b] \times \boldsymbol{R}, \int_{\boldsymbol{R}} \phi(t, v) d \sigma_{t}(v)$ is a measurable function of $t$ and

$$
\int_{a}^{b}\left[\int_{R} \phi(t, v) d \sigma_{t}(v)\right] d t=\int_{(a, b] \times R} \phi(t, v) d \mu(t, v) .
$$

First let $\phi(t, v)=\chi_{E}(t, v)$ where $E$ is in $\mathscr{B}((a, b] \times \boldsymbol{R})$. Then

$$
\int_{R} \chi_{E}(t, v) d \sigma_{t}(v)=\int_{R} \chi_{E^{(t)}}(v) d \sigma_{t}(v)=\sigma_{t}\left(E^{(t)}\right)
$$

which is measurable as a function of $t$ by assumption. Also

$$
\begin{array}{r}
\int_{a}^{b}\left[\int_{R} \chi_{E}(t, v) d \sigma_{t}(v)\right] d t=\int_{a}^{b} \sigma_{t}\left(E^{(t)}\right) d t=\mu(E) \\
=\int_{(a, b] \times \mathbf{R}} \chi_{E}(t, v) d \mu(t, v) \text { as desired. }
\end{array}
$$

The result now follows easily for simple functions $\phi$ by linearity. For $\phi$ a bounded measurable function, take a sequence $\left\{\phi_{n}\right\}$ of simple functions such that $\left\|\phi_{n}\right\|_{\infty} \leqq\|\phi\|_{\infty}$ and $\phi_{n}$ converges to $\phi$ uniformly. By the Dominated Convergence Theorem, 


$$
\int_{R} \phi(t, v) d \sigma_{t}(v)=\lim _{n \rightarrow \infty} \int_{R} \phi_{n}(t, v) d \sigma_{t}(v)
$$

But $\int_{R} \phi_{n}(t, v) d \sigma_{t}(v)$ is a measurable function of $t$ for each $n$ and so $\int_{R} \phi(t, v) d \sigma_{t}(v)$ is a measurable function of $t$. Next note that

$$
\left|\int_{R} \phi_{n}(t, v) d \sigma_{t}(v)\right| \leqq \int_{R}\|\phi\|_{\infty} d\left|\sigma_{t}\right|(v)=\|\phi\|_{\infty}\left\|\sigma_{t}\right\| \leqq\|\phi\|_{\infty} h(t)
$$

for every $n$. This justifies the second of the three uses of the Dominated Convergence Theorem in the following string of equalities.

$$
\begin{aligned}
\int_{(a, b] \times R} \phi(t, v) d \mu(t, v) & =\lim _{n \rightarrow \infty} \int_{(a, b] \times R} \phi_{n}(t, v) d \mu(t, v) \\
& =\lim _{n \rightarrow \infty} \int_{a}^{b}\left[\int_{\boldsymbol{R}} \phi_{n}(t, v) d \sigma_{t}(v)\right] d t \\
& =\int_{a}^{b}\left[\lim _{n \rightarrow \infty} \int_{R} \phi_{n}(t, v) d \sigma_{t}(v)\right] d t \\
& =\int_{a}^{b}\left[\int_{R} \lim _{n \rightarrow \infty} \phi_{n}(t, v) d \sigma_{t}(v)\right] d t \\
& =\int_{a}^{b}\left[\int_{R} \phi(t, v) d \sigma_{t}(v)\right] d t \text { as desired. }
\end{aligned}
$$

For each $x, \exp \{i v x(t)\}$ is a bounded Borel measurable function of $(t, v)$. Hence $f(x)=\int_{a}^{b}\left[\int_{R} \exp \{i v x(t)\} d \sigma_{t}(v)\right] d t$ equals

$$
\int_{(a, b] \times \boldsymbol{R}} \exp \{i v x(t)\} d \mu(t, v)
$$

as desired.

We now know that $F(x)=\exp \left\{\int_{a}^{b} \theta(t, x(t)) d t\right\}=\sum_{n=0}^{\infty}(1 / n !) f^{n}(x)$ is in $S$ where $f$ is given by (3.5). Further we know from the proofs of Theorem 1 and Lemma 3 that $f$ is associated with the measure $\sigma=\mu_{\circ} \Phi^{-1}$ where $\mu$ is the measure from the preceding theorem. Because convolution is taken over into pointwise multiplication by the map from $\mathscr{C}\left(L_{2}\right)$ onto $S$, the measure $(1 / n !) \sigma * \cdots * \sigma$ ( $n$ convolutions) is associated with $(1 / n !) f^{n}$. Now $\|(1 / n !) \sigma * \cdots * \sigma\| \leqq(1 / n !)\|\sigma\|^{n}$ and so, of course, $\sum_{n=1}^{\infty}(1 / n !)\|\sigma * \cdots * \sigma\|<\infty$. Under these conditions, as noted earlier, the analytic Feynman integral of the sum is the sum of the analytic Feynman integrals, and so we have

$$
\int_{C[a, b]}^{\mathrm{anf}_{q}} F(x) d m(x)=\sum_{n=0}^{\infty} \frac{1}{n !} \int_{C[a, b]}^{\operatorname{anf}_{q}} f^{n}(x) d m(x) .
$$

Hence we will have a formula for $\int_{C[a, b]}^{\operatorname{anf}_{q}} F(x) d m(x)$ as a series if we obtain a formula for $(1 / n !) \int_{C[a, b]}^{\mathrm{anf} q} f^{n}(x) d m(x)$. We will obtain such a 
formula below, expressing $(1 / n !) \int_{C[a, b]}^{\mathrm{anf}_{q}} f^{n}(x) d m(x)$ as an integral over a finite-dimensional space.

For now, let $\lambda>0$. We wish to calculate

$$
\frac{1}{n !} \int_{C[a, b]} f^{n}\left(\lambda^{-1 / 2} x\right) d m(x) \text {. }
$$

We know from the discussion of the preceding theorem that $f(x)=$ $\int_{(a, b] \times R} \exp \{i v x(t)\} d \mu(t, v)$ where $\mu$ is a $C$-valued Borel measure on $(a, b] \times R$ and so, $|f(x)| \leqq\|\mu\|$ for all $x$. Certainly then the Wiener integral above exists.

We will use the following notation:

$$
\Delta_{n}=\left\{\left(t_{1}, \cdots, t_{n}\right) \text { in }[a, b]^{n}: a=t_{0}<t_{1}<\cdots<t_{n} \leqq b\right\} .
$$

LEMma 4. For $\lambda>0$,

$$
\begin{gathered}
{\left[\frac{\lambda}{2 \pi\left(t_{j}-t_{j-1}\right)}\right]^{1 / 2} \int_{R} \exp \left\{\frac{-\lambda\left(u_{j}-u_{j-1}\right)^{2}}{2\left(t_{j}-t_{j-1}\right)}+i u_{j} \omega\right\} d u_{j}} \\
=\exp \left\{i u_{j-1} \omega-\frac{\left(t_{j}-t_{j-1}\right)}{2 \lambda} \omega^{2}\right\} .
\end{gathered}
$$

The lemma follows from the change of variables

$$
z=\left[\frac{\lambda}{t_{j}-t_{j-1}}\right]^{1 / 2}\left(u_{j}-u_{j-1}\right)
$$

and from the fact that $\exp \left\{-z^{2} / 2\right\}$ is its own Fourier transform; i.e.,

$$
(2 \pi)^{-1 / 2} \int_{R} \exp \left\{-z^{2} / 2+i z \omega\right\} d z=\exp \left(-\omega^{2} / 2\right) .
$$

The lemma actually holds for $\lambda$ in $\boldsymbol{C}^{+}$, but we only need it for $\lambda>0$. For $\lambda=-i q(q \neq 0)$, the right hand side above makes sense, but the integral does not exist as a Lebesgue integral.

Now we can write

$$
\begin{aligned}
\frac{1}{n !} \int_{C[a, b]} & f^{n}\left(\lambda^{-1 / 2} x\right) d m(x) \\
& =\frac{1}{n !} \int_{C[a, b]}\left[\prod_{j=1}^{n} \int_{a}^{b} \theta\left(t_{j}, \lambda^{-1 / 2} x\left(t_{j}\right)\right) d t_{j}\right] d m(x) \\
& =\frac{1}{n !} \int_{C[a, b]} \int_{[a, b]^{n}}\left[\prod_{j=1}^{n} \theta\left(t_{j}, \lambda^{-1 / 2} x\left(t_{j}\right)\right)\right] d t_{1} \cdots d t_{n} d m(x) \\
& =\frac{n !}{n !} \int_{C[a, b]} \int_{\Delta_{n}}\left[\prod_{j=1}^{n} \theta\left(t_{j}, \lambda^{-1 / 2} x\left(t_{j}\right)\right)\right] d \vec{t} d m(x) \\
& =\int_{\Delta_{n}} \int_{C[a, b]}\left[\prod_{j=1}^{n} \theta\left(t_{j}, \lambda^{-1 / 2} x\left(t_{j}\right)\right)\right] d m(x) d \vec{t}
\end{aligned}
$$


where the use of the Fubini Theorem is justified since

$$
\begin{aligned}
\int_{C[a, b]} & \int_{\Delta_{n}} \prod_{j=1}^{n}\left|\theta\left(t_{j}, \lambda^{-1 / 2} x\left(t_{j}\right)\right)\right| d \vec{t} d m(x) \\
& =\int_{C[a, b]} \int_{\Delta_{n}} \prod_{j=1}^{n}\left|\int_{R} \exp \left\{i \lambda^{-1 / 2} x\left(t_{j}\right) v_{j}\right\} d \sigma_{t_{j}}\left(v_{j}\right)\right| d \vec{t} d m(x) \\
& \leqq \int_{C[a, b]} \int_{\Delta_{n}} \prod_{j=1}^{n} h\left(t_{j}\right) \overrightarrow{d t} d m(x) \\
& =\frac{1}{n !} \int_{C[a, b]} \int_{[a, b]^{n}} \prod_{j=1}^{n} h\left(t_{j}\right) d \vec{t} d m(x)<\infty .
\end{aligned}
$$

By applying a basic Wiener integration formula and then making a simple change of variables, we see that the last expression in (3.6) equals

$$
\begin{aligned}
& \int_{\Delta_{n}} \lambda^{n / 2}\left[(2 \pi)^{n}\left(t_{1}-a\right) \cdots\left(t_{n}-t_{n-1}\right)\right]^{-1 / 2} \\
& \int_{R^{n}}\left[\prod_{j=1}^{n} \theta\left(t_{j}, u_{j}\right)\right] \exp \left\{\frac{-\lambda}{2} \sum_{j=1}^{n} \frac{\left(u_{j}-u_{j-1}\right)^{2}}{t_{j}-t_{j-1}}\right\} d \vec{u} d \vec{t} \\
& =\int_{\Delta_{n}} \lambda^{n / 2}\left[(2 \pi)^{n}\left(t_{1}-a\right) \cdots\left(t_{n}-t_{n-1}\right)\right]^{-1 / 2} \\
& \int_{R^{n}}\left[\prod_{j=1}^{n} \int_{R} \exp \left\{i u_{j} v_{j}\right\} d \sigma_{t_{j}}\left(v_{j}\right)\right] \exp \left\{\frac{-\lambda}{2} \sum_{j=1}^{n} \frac{\left(u_{j}-u_{j-1}\right)^{2}}{t_{j}-t_{j-1}}\right\} d \vec{u} d \vec{t} .
\end{aligned}
$$

One can easily justify integrating first with respect to the $u_{j}$ 's. Doing this and applying Lemma $4 n$ times, we obtain

$$
\begin{gathered}
\int_{\Delta_{n}} \int_{R^{n}} \exp \left\{-\frac{1}{2 \lambda}\left[\sum_{l=1}^{n} \sum_{j=1}^{l}\left(2-\delta_{j, l}\right) v_{j} v_{l}\left(t_{j}-a\right)\right]\right\} \\
\times d \sigma_{t_{1}}\left(v_{1}\right) \cdots d \sigma_{t_{n}}\left(v_{n}\right) d \vec{t} \equiv g_{n}(\lambda) .
\end{gathered}
$$

So for $\lambda>0,(1 / n !) \int_{C[a, b]} f^{n}\left(\lambda^{-1 / 2} x\right) d m(x)=g_{n}(\lambda)$.

Note next that the integral giving $g_{n}(\lambda)$ in (3.7) exists for $\operatorname{Re} \lambda \geqq 0(\lambda \neq 0)$ since the integrand is bounded by 1 and

$$
\int_{\Delta_{n}} h\left(t_{1}\right) \cdots h\left(t_{n}\right) d \vec{t}<\infty
$$

Because of this it is not difficult to argue in the usual way via Morera's Theorem, the Fubini Theorem and the Cauchy Integral Theorem that $g_{n}(\lambda)$ is analytic in $C^{+}$. Also it is not hard to see that $g_{n}(\lambda) \rightarrow g_{n}(-i q)$ as $\lambda \rightarrow-i q$ through $C^{+}$. Hence

$$
\int_{C[a, b]}^{\operatorname{anf}_{q}} \frac{1}{n !} f^{n}(x) d m(x)
$$

exists and equals $g_{n}(-i q)$. 
We summarize in the following theorem.

THEOREM 2. Under the hypotheses of Theorem 1 we have the formula

$$
\begin{aligned}
\int_{C[a, b]}^{\operatorname{anf}_{q}} F(x) d m(x)=1 \\
+\sum_{n=1}^{\infty} \int_{\Delta_{n}} \int_{R^{n}} \exp \left\{\frac{1}{2 q i}\left[\sum_{l=1}^{n} \sum_{j=1}^{l}\left(2-\delta_{j, l}\right) v_{j} v_{l}\left(t_{j}-a\right)\right]\right\} \\
\quad \times d \sigma_{t_{1}}\left(v_{1}\right) \cdots d \sigma_{t_{n}}\left(v_{n}\right) d \vec{t}
\end{aligned}
$$

where $F$ is given by (1.1).

We end this paper by remarking that the work done above for the exponential of $\int_{a}^{b} \theta(t, x(t)) d t$ can be carried out just as well for other analytic functions of $\int_{a}^{b} \theta(t, x(t)) d t$.

Note added in proof. The conditions on $\theta$ in Theorem 1 can be improved slightly to conditions which are equivalent but formally weaker. This is discussed briefly in Corollary 4 of "Notes on the Feynman integral I", to appear in the J. of Functional Analysis.

\section{REFERENCES}

1. S. Albeverio and R. Høegh-Krohn, Mathematical Theory of Feynman Path Integrals, Springer Verlag Lecture Notes in Mathematics, 523 (1976).

2. R. H. Cameron and D. A. Storvick, Some Banach algebras of analytic Feynman integrable functionals, Proceedings of 7 th international conference on analytic functions, Kozubnik, Poland, Springer Verlag Lecture Notes in Mathematics, 798 (1980), 18-67.

3. P. Halmos, Measure Theory, Van Nostrand, Princeton, New Jersey, 1950.

4. G. W. Johnson and D. L. Skoug, Scale-invariant measurability in Wiener space, Pacific J. Math., 83 (1979), 157-176.

5. - A Banach algebra of Feynman integrable functionals with application to an integral equation formally equivalent to Schroedinger's equation, J. Functional Analysis, 12 (1973), 129-152.

6. J. Kuelbs and V. Mandrekar, Harmonic analysis on F-spaces with a basis, Trans. Amer. Math. Soc., 169 (1972), 113-152.

7. R. E. A. C. Paley, N. Wiener and A. Zygmund, Notes on Random Functions, Math. Zeit., 37 (1933), 647-688.

Received October 26, 1979. The first author gratefully acknowledges the support of the U. of Nebraska as well as the hospitality of the Mathematisches Institut, Universität Erlangen-Nürnberg and especially the kind help of Professor Kölzow and Dr. Tischer.

Mathematisches Institut, Universität ERLangen-Nürnberg

ERLANGEN 8520, BRD (Visiting 1979/80)

AND

University of Nebraska-Lincoln

Lincoln, NE 68588 


\title{
PACIFIC JOURNAL OF MATHEMATICS
}

\section{EDITORS}

\author{
DoNALD BABBITT (Managing Editor) \\ University of California \\ Los Angeles, CA 90024 \\ Hugo RossI \\ University of Utah \\ Salt Lake City, UT 84112 \\ C. C. MOORE and ANDrew OGG \\ University of California \\ Berkeley, CA 94720
}

J. DugundJI

Department of Mathematics

University of Southern California

Los Angeles, CA 90007

R. FINN and J. MILGRAM

Stanford University

Stanford, CA 94305

\section{ASSOCIATE EDITORS}
R. Arens
E. F. BeCKenbaCH
B. H. NeumanN
F. WOLF
K. YoSHIDA

\section{SUPPORTING INSTITUTIONS}

UNIVERSITY OF ARIZONA

UNIVERSITY OF BRITISH COLUMBIA

CALIFORNIA INSTITUTE OF TECHNOLOGY

UNIVERSITY OF CALIFORNIA

MONTANA STATE UNIVERSITY

UNIVERSITY OF NEVADA, RENO

NEW MEXICO STATE UNIVERSITY

OREGON STATE UNIVERSITY

\author{
UNIVERSITY OF OREGON \\ UNIVERSITY OF SOUTHERN CALIFORNIA \\ STANFORD UNIVERSITY \\ UNIVERSITY OF HAWAII \\ UNIVERSITY OF TOKYO \\ UNIVERSITY OF UTAH \\ WASHINGTON STATE UNIVERSITY \\ UNIVERSITY OF WASHINGTON
}

The Supporting Institutions listed above contribute to the cost of publication of this Journal, but they are not owners or publishers and have no responsibility for its content or policies.

Mathematical papers intended for publication in the Pacific Journal of Mathematics should be in typed form or offset-reproduced, (not dittoed), double spaced with large margins. Please do not use built up fractions in the text of the manuscript. However, you may use them in the displayed equations. Underline Greek letters in red, German in green, and script in blue. The first paragraph or two must be capable of being used separately as a synopsis of the entire paper. Please propose a heading for the odd numbered pages of less than 35 characters. Manuscripts, in triplicate, may be sent to any one of the editors. Please classify according to the scheme of Math. Reviews, Index to Vol. 39. Supply name and address of author to whom proofs should be sent. All other communications should be addressed to the managing editor, or Elaine Barth, University of California, Los Angeles, California, 90024.

50 reprints to each author are provided free for each article, only if page charges have been substantially paid. Additional copies may be obtained at cost in multiples of 50 .

The Pacific Journal of Mathematics is issued monthly as of January 1966. Regular subscription rate: $\$ 102.00$ a year $(6$ Vols., 12 issues). Special rate: $\$ 51.00$ a year to individual members of supporting institutions.

Subscriptions, orders for numbers issued in the last three calendar years, and changes of address shoud be sent to Pacific Journal of Mathematics, P.O. Box 969, Carmel Valley, CA 93924, U.S.A Old back numbers obtainable from Kraus Periodicals Co., Route 100, Millwood, NY 10546.

\section{PUBLISHED BY PACIFIC JOURNAL OF MATHEMATICS, A NON-PROFIT CORPORATION}

Printed at Kokusai Bunken Insatsusha (International Academic Printing Co., Ltd.). 8-8, 3-chome, Takadanobaba, Shinjuku-ku, Tokyo 160, Japan. 


\section{Pacific Journal of Mathematics}

Vol. 93, No. $2 \quad$ April, 1981

Ilya Eugene Blum and Srinivasa Swaminathan, Continuous selections and

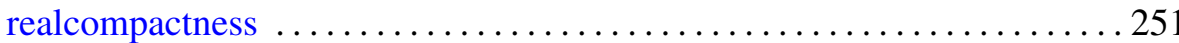

Lawrence James Brenton, Differentiably $k$-normal analytic spaces and extensions of holomorphic differential forms $\ldots \ldots \ldots \ldots \ldots \ldots \ldots 261$

Jo-Ann Deborah Cohen, Topologies on the ring of integers of a global field ................................................... 269

Robert Jay Daverman, Detecting the disjoint disks property $\ldots \ldots \ldots \ldots 277$

Edmund H. Feller, Rings where the annihilators of $\alpha$-critical modules are prime ideals ....................................... 299

Richard Elam Heisey and Henryk Torunczyk, On the topology of direct

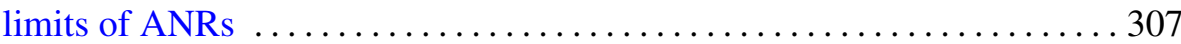

Gerald William Johnson and David Lee Skoug, Notes on the Feynman

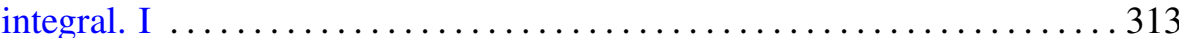

Michael S. Keane and Stuart Jay Sidney, Distinguishing a plane curve

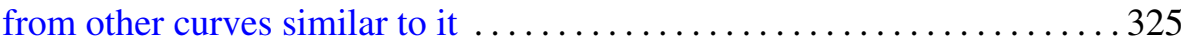

Leonid A. Luxemburg, On compact metric spaces with noncoinciding

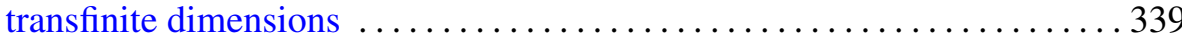

Chun Ming Ma, A uniqueness theorem for Navier-Stokes equations . . . . . . 387

Donald J. Newman and Theodore Joseph Rivlin, A characterization of the weights in a divided difference . . . . . . . . . . . . . . . . . . . 407

Marc Aristide Rieffel, $C^{*}$-algebras associated with irrational rotations $\ldots .415$

Kichi-Suke Saito, Invariant subspaces for finite maximal subdiagonal algebras

Frederic W. Shultz, Dual maps of Jordan homomorphisms and ${ }^{*}$-homomorphisms between $C^{*}$-algebras $\ldots \ldots \ldots \ldots$

Vsevolod Alekseevich Solonnikov, On the solvability of boundary and initial-boundary value problems for the Navier-Stokes system in domains with noncompact boundaries

Tavan Thomas Trent, New conditions for subnormality

L. E. Ward, Extending Whitney maps ..................

Leslie Wilson, Jets with regular zeros

Sergio Eduardo Zarantonello, The sheaf of $H^{p}$-functions in product 